\title{
Research on Online Education Teacher Evaluation Model based on Opinion Mining
}

\author{
LI Caiqiang \\ Online and Continuing College \\ Southwest University \\ Beibei, Chongqing, China, 023-68254525 \\ licaiq@swu.edu.cn
}

\author{
Ma Junming \\ School of Foreign Language \\ Southwest University \\ Beibei, Chongqing, China, 023-68254669 \\ Lunar_ma@swu.edu.cn
}

\begin{abstract}
This paper analyzes the current common teacher evaluation methods, and points out the shortcoming of current methods of using the rubrics: they rely on the fixed rubric rules, and take less into account students' opinion text published in the LMS (learning management system). The paper provides an online education teacher evaluation model based on opinion mining. The model collects opinion texts in the LMS by using web crawler, and processes them by using topic extraction and sentiment orientation classification, etc. And thus the model gets an overall evaluation of each teacher. This processing can primarily run automatically and enhance efficiency and effect of the teacher evaluation.
\end{abstract} Mining

Keywords-Online Education; Teacher Evaluation; Opinion

\section{CURRENT CONDITION OF ONLINE EDUCATION TEACHER EVALUATION}

Teachers, who are in charge of designating and providing teaching materials and providing learning supporting services to online students, play an important role in the quality of online education. Comprehensive and reliable teacher evaluation methods can increase enthusiasm, initiative and creativity of teachers [1]. Current online education teacher evaluation is always built according to traditional teacher evaluation methods. National Standards for Quality Online Teaching [2] propose 13 guidelines to evaluate teachers. For example, "The teacher meets the professional teaching standards established by a state-licensed agency or the teacher has academic credentials in the field in which he or she is teaching" and "The teacher has the prerequisite technology skills to teach online". Yang and his team research on the Radio and TV University's teacher evaluation and propose "In the current teacher evaluation guidelines, a teacher is evaluated according to his teaching load, such as how many courses the teacher teaches, how well these courses are taught, whether the teacher has a teaching accident, etc”. ”But students' scores and students' evaluation of teaching and teaching materials have nothing to do with the evaluation of a teacher" [3]. There are some public websites where students can comment their teachers online, for example, a Chinese website www.pinglaoshi.com, an American website www.ratemyprofessors.com, etc. These websites allow students

Supported by "the Fundamental Research Funds for the Central Universities”. No: XDJK2012C030 to give comments on their teachers according to some fixed indexes.

Most of the current teacher evaluation research is considering how to construct an evaluation index system, evaluating teachers from the index system with different subscores, achieving the general evaluation from the total scores. The shortcomings of those researches are that the evaluation is confined to some fixed evaluating indexes. In fact, students, who act as the most important users of online education system, comment teachers online whenever and wherever possible. For example, after his or her homework is graded online, a student writes comments "the teacher graded homework so earnest", or a student comment "the teacher grades homework so slowly" when he or she finds homework not being graded after he or she finished homework some days later, or a student comments "thanks for the teacher's detailed answers" in the course forum, etc. All of these comments about teaching services and teaching materials are published by students who enjoy these services and materials. Their comments should have occupied greater weight in the teacher evaluation because they are more authentic and important. But these subjective comments are less talked about in the current studies.

The paper proposes a teacher evaluation method based on opinion mining, which crawls student comments on teachers to form a teacher evaluation repository automatically. The method classifies these comments to positive or negative category with opinion mining methods and points out which is good or not good about teachers work. An overall positive or negative evaluation of a teacher is given out from students' perspective.

\section{INTRODUCTION TO OPINION MINING}

Opining mining or sentiment analysis is mining data from subject texts. The Internet text can be divided into two categories, objective text and subjective text. Objective text is the objective description about some facts and events. Subjective text is the description of people's views or attitudes towards events or people. With the rapid development of Internet, especially wide application of web 2.0, people post many texts with their views or attitudes in blogs, forums, electronic business websites. It will get lots of valuable information through the analysis and classification of these texts. For example, customers always remark online after they bought some goods in the electronic business websites. These 
remarks always include customers' description of the goods' advantages and shortcomings. Through collecting and mining these subjective texts we can analyze the products' advantages and shortcomings in customers' perspective. With the results, sellers can improve the product and potential customers can get useful reference when making a purchase.

Kim and Hovy describe an opinion as a quadruple[ Topic, Holder, Claim, Sentiment] in which the Holder believes a Claim about the Topic, and in many cases associates a Sentiment, such as good or bad, with the belief [5].

Opinion mining operation always includes the following four steps:

a) Topic Extraction: Identifying a topic and assigning domain related ontology concepts.

b) Holder Identification: Identifying opinion owner.

c) Claim Selection: Confirming the scope of opinion and eliminating objective claim.

d) Sentiment Analysis: Judging the claim's semantic orientation or polarity.

Some subjective texts as follows, "I feel IPAD2 is very good, higher screen resolution, quicker running speed, easy to carry." In this text, the opinion holder is "I", the topic is "IPAD2", "screen resolution”, "running speed”, "carrying” are the properties of the claim. "Higher”, "quicker”, "easy” are the sentiment of the claim.

In a teaching and learning system, opinion holders are always students who use learning resources and enjoy learning support services. These students can be recognized as equal opinion holders. So we do not take into account the opinion holder identification, but yet emphasize Topic Extraction, Polarity dictionary construction and Sentiment Analysis by opinion mining method.

\section{OPINION MINING BASED TEACHER EVALUATION MODEL}

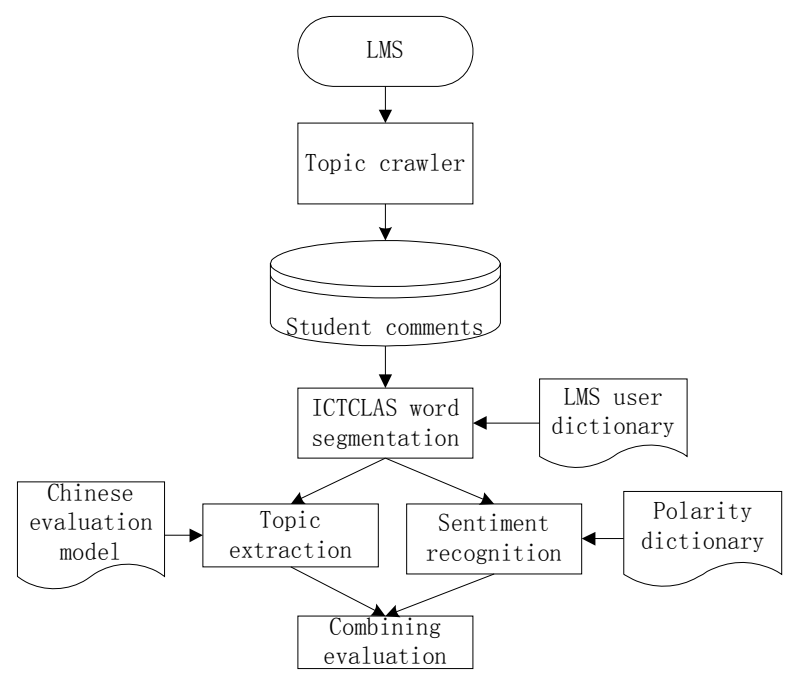

Figure 1. OMTEM

A model named Opinion Mining based Teacher Evaluation Model (OMTEM) is proposed based on teacher evaluation and opinion mining theory. Figure 1 is about OMTEM. In OMTEM, firstly, topic crawler crawls the comments on teachers from the online education system, such as from the column of course forum, and student blogs, etc. A teacher comments repository is established with these crawled comments. Next, OMTEM uses word segmentation technology to group these comments. And then these comments will be processed by topic extraction and sentiment recognition. At last, a general evaluation of a teacher will be formed.

The paper focuses on Chinese word segmentation, topic extraction, sentiment recognition of OMTEM.

\section{WORD SEGMENTATION}

Chinese word segmentation is one of the bases of web mining. Computer can deal with Chinese words correctly and recognize their right meanings only when these words are split appropriately. So, we should finish word segmentation and speech tagging of the Chinese sentence in order to execute topic extraction from the student comments repository. There are some Chinese word segmentation technology, for example, Chinese word segmentation engine SCWS based on word frequency developed by Hightman, open source Paoding component based on java language, ICTCLAS developed by Institute of Computing Technology Chinese Academy of Science[7], etc.

OMTEM utilizes the widely-used word segmentation technology ICTCLAS to realize word segmentation and speech tagging. ICTCLAS has an advantage that it can support user defined dictionary. Word segmentation results will be more accurate in special environment by using the user defined dictionary. Take the following as an example, if we define a new word 'thesis defense' in the education domain., 'thesis defense' will be recognized as a word, not two words with 'thesis' and 'defense' after segmenting. In order to get the better result of online education domain we define an online education administration dictionary. With the online education administration dictionary ICTCLAS can segment the Chinese words better in online education domain. For example, using ICTCLAS and the dictionary “张老师论文指导很认真” will be segmented to '张/q 老师/n 论文指导/n 很/d 认真/a'. In the result, nouns are marked as $n$, verbs as $\mathrm{v}$, adverbs as $\mathrm{d}$, adjective as a, etc. Topic extraction will be done after word segmentation is fulfilled with comment sentences.

\section{TOPIC EXTRACTION}

Topic extraction from the student comments, referring to different aspects that students comment teachers. We call these different aspects as characteristic values in opinion mining process. There are a variety of topic extraction methods, mainly divided into artificial definition method and automatic extraction method. The paper utilizes the automatic extraction method referring to Zhu \& Li's method[8]. Zhu \& Li realize the topic extraction based on Chinese grammar model. They summarize the common grammar patterns for Chinese opinion expressions based on a Chinese product reviews training corpus, see Table 1. 
TABLE 1. COMMON GRAMMAR PATTERNS FOR CHINESE REVIEWS

\begin{tabular}{c|l}
\hline \multicolumn{1}{c|}{ Pattern } & Pattern definition \\
\hline pattern 1 & Noun+ adj. \\
\hline pattern 2 & Noun+ad.+adj. \\
\hline pattern 3 & Adj.+的+noun \\
\hline
\end{tabular}

We use the grammar patterns in Table 1 to realize topic extraction in student comments repository, which is dealt with word segmentation. We mainly choose nouns as the topic in the topic extraction. These topics are stored in a topic candidate sets and listed in order of their occurrence frequency from high to low. We choose the high frequency topics as a teacher's characteristic values, see Table 2 .

TABLE 2. TOPIC CANDIDATE

\begin{tabular}{c|l}
\hline Topic candidate & Term frequency \\
\hline topic 1 & TF1 \\
\hline topic 2 & TF2 \\
\hline topic 3 & TF3 \\
\hline$\ldots$ & $\ldots$ \\
\hline
\end{tabular}

\section{POLARITY WORDS DICTIONARY} CONSTRUCTION

Polarity words dictionary is constructed as selecting some polarity words from HowNet[9]. The sentiment words are categorized into Chinese words and English words in the HowNet. We choose 200 positive words and 160 negative words from HowNet to do experiment. Considering the popular Internet language, we add some Internet words to the polarity dictionary. For example, “稀” same to “喜欢”, “顶” same to "agree”, etc. We do not consider negative \& turning vocabulary in the early phase of the experiment, see Table 3.

TABLE 3. POLARITY WORDS DICTIONARY

\begin{tabular}{l|c|l}
\hline No. & Polarity words & polarity \\
\hline 1 & 精彩 & 2 \\
\hline 2 & 恶劣 & -2 \\
\hline$\ldots$. & $\ldots$ & $\ldots$ \\
\hline$m$ & 顶 & 1 \\
\hline$m+1$ & 稀饭 & 1 \\
\hline
\end{tabular}

The words are divided into negative and positive words in HowNet. But it does not indicate the words polarity strength.
The paper sets the polarity scope $(-2,2)$. “-” means negative, “+” means positive, larger absolute value, stronger polarity.

If sentiment words of subjective text are not in the polarity words dictionary, the paper uses pointwise mutual information (PMI) method to judge the polarity of it, combined with the Internet search engine.

$$
\operatorname{PMI}(x, y)=\log \frac{p(x, y)}{p(x) p(y)}
$$

$\mathrm{P}(\mathrm{x}, \mathrm{y})$ indicates that search engine returns records number recognizing $\mathrm{x}$ and $\mathrm{y}$ as a combining keyword. $\mathrm{p}(\mathrm{x})$ and $\mathrm{p}(\mathrm{y})$ indicate that search engine returns records number recognizing $\mathrm{x}$ and $\mathrm{y}$ as separate keywords. Here we select two seed words. One is “优美”, the other is “丑恶”. If w is a sentiment word which is not in the polarity words dictionary we calculate w's PMI with ‘优美’ and ‘丑恶’ separately.

$$
\text { New word polarity=PMI(w, 优美) }-\mathrm{PMI}(\mathrm{w} \text {, 丑恶) }
$$

If new word polarity is bigger than zero then $\mathrm{w}$ is a positive sentiment word, if new word polarity is less than zero then $\mathrm{w}$ is a negative sentiment word.

\section{EXPERIMENT}

\section{A. Collecting comments data}

In the experiment, we choose a Chinese online education college's LMS to testify the effect of online education teacher evaluation. The college has over 50,000 online students. There are lots of student comments on teachers in the columns of course forums, student bolgs and resource repository of college's LMS.

We choose Myspiders[11] to crawl student comments from LMS, and process the crawling results to triples like this, <course name, teacher, student comments>, some data is supplemented with reference to LSM's database.

\section{B. Charateristic value selection}

We get over 100 candidate topics from data collected by using Chinese grammar patterns. We choose candidate topics of whice term frequency are bigger than 5 as teacher evaluation characteristic values. We get 35 characteristic values.

TABLE 4. CHARACTERISTIC VALUE

\begin{tabular}{c|l|l}
\hline $\begin{array}{c}\text { Characteristic } \\
\text { value }\end{array}$ & $\begin{array}{l}\text { Term } \\
\text { frequency }\end{array}$ & description \\
\hline 作业批改 & 89 & $\begin{array}{l}\text { the attitude of teacher grading } \\
\text { homework }\end{array}$ \\
\hline 答疑 & 72 & $\begin{array}{l}\text { the condition of teacher answering } \\
\text { question in forum }\end{array}$ \\
\hline 论文指导 & 47 & $\begin{array}{l}\text { The condition of teacher reviewing } \\
\text { thesis }\end{array}$ \\
\hline$\ldots$ & $\ldots$ & $\ldots$ \\
\hline 普通话 & 5 & teacher's mandarin Chinese level \\
\hline
\end{tabular}




\section{Judging polarity of teacher evaluation}

We mainly choose adj. as candidate polarity words after student comments being processed with word segmentation, and judge the polarity of student comments' characteristic values in reference to polarity word dictionary. We sum the polarity of each teacher's comments to a value $x$, count the student comments of each teacher to a value $y$. Then we use the formula $\mathrm{z}=\mathrm{x} / \mathrm{y}$. If $\mathrm{z}>0$, then the teacher evaluation's value is positive. It means most students are satisfied with the teacher's work, vice versa. teacher:

For example, there are four student comments on a

1) “老师的资源很好”. Its polarity value is 1 .

2) “答疑很耐心”. Its polarity value is 1 .

3) “普通话水平比较差”. Its polarity value is -1 .

4) “我很稀饭老师的资源”. Its polarity value is 1 . Here $\mathrm{x}=1+1-1+1=2, \mathrm{y}=4$, then $\mathrm{z}=\mathrm{x} / \mathrm{y}=2 / 4=0.5$.

0.5 is bigger than zero, indicating that most students are satisfied with the teacher's work. The teacher's evaluation is positive.

\section{CONCLUSION}

The paper proposes a new teacher evaluation method based on opinion mining named OMTEM, considering the students' online comments in the LMS. Nowadays, the web 2.0 application is widely used. Combining the students' comments, OMTEM is direct, useful, and persuasive in teacher evaluation. It can make up the shortcomings of firmed index in traditional teacher evaluation.
However, further work need be carried out on characteristic value recognition and polarity word dictionary in order to achieve a better result.

\section{REFERENCES}

[1] Teacher Evaluation of online education, [EB/OL] . http://www.chinaret.com/user/article.aspx?cid=417200d2-f785-47ddbc49-318d3a95b764 ,2009.5.26/2011.6.18.

[2] National Standards for Quality Online Teaching, [EB/OL] . http://www.inacol.org/research/nationalstandards/NACOL\%20Standards \%20Quality\%20Online\%20Teaching.pdf, 2010.8.1/2011.6.18.

[3] Yang Tingting, Yan Fu'an. The Comparison of teacher Teaching Management in Distance Education between China and America[J]. Open Education Research.2006(3), 35-38.

[4] Hou Feng, Wang Chuanting, Li Guohui. Survey on the Opinion Mining,Summarization and Retrieval[J]. Computer Science. 2009(7), 1519.

[5] S.-M. Kim and E. Hovy. Determining the Sentiment of Opinions [A]. Proceedings of the International Conference on Computational Linguistics (COLING2004) [C]. Geneva, Switzerland:2004,1367-1373.

[6] Yao Tian, Cheng Xiwen. A Survey of Opinion Mining for Texts[J]. Journal of Chinese Information Processing.2008(3), 71-80.

[7] Institute of Computing Technology Chinese Academy of Science, ICTCLAS[EB/OL]. http://ictclas.org/index.html,2011.8.1/2012.2.10

[8] Zhu Zhengyu, Li Cunqing, Zhang Peng. Topic words and Opinion Words Extraction From Chinese Product Reviews Based on Syntax Pattern[J]. Journal of Chongqing University of Technology (Natural Science ).2010(5), 86-90

[9] HowNet, [EB/OL]. http://www.keenage.com/zhiwang/c_zhiwang.html, 2003.1.1/2012.2.1.

[10] Pointwise mutual information, [EB/OL]. http://en.wikipedia.org/wiki/Pointwise_mutual_information,2012.2.5

[11] Myspiders, [EB/OL]. http://Myspiders.informatics.indiana.edu,2003.1.1/2012.2.1 